\title{
Hospitalization rates and resource utilization of schizophrenic patients switched from oral antipsychotics to aripiprazole-depot in Germany
}

\author{
Christoph Potempa* (i) and Reinhard Rychlik
}

\begin{abstract}
Objective: Examine cost-driving factors of schizophrenia in Germany for patients prior- and post-switch from an oral antipsychotic therapy to aripiprazole-depot and perform a budget impact analysis (BIA) referring to the context of German health care.

Methods: A single-armed, retrospective, non-interventional pre-post comparison study with 132 patients to compare the total psychiatric hospitalization rates and the associated costs of both, the treatment with oral antipsychotics and aripiprazole-depot. The BIA was performed to compare both treatment periods with respect to health-related costs. A subsequent univariate sensitivity analysis examined the robustness of the results.

Results: After switching the treatment to aripiprazole-depot, the total psychiatric hospitalization rates for the 6-month treatment period were significantly $(p<0.001)$ lower $(14 \%)$ compared to the hospitalization rates when treated with oral antipsychotics (55.1\%). 18.2\% of the patients reported to be employed, with $29.2 \%$ having work incapacities. The mean number of schizophrenia episodes was 2.58 episodes per patient during the oral-antipsychotic treatment compared to 0.41 episodes per patient during the aripiprazole-depot phase $(p<0.001)$. The treatment with aripiprazole-depot also significantly reduced the mean number of hospitalizations per patient $(0.63$ to $0.16, p<0.001)$ and the mean number of hospitalized days ( 27.39 to $5.56, p<0.001)$ compared to the oral antipsychotic treatment. Additionally a significant reduction of the mean stay in day-clinics and psychiatric institute ambulances (PIAs) was observed (46.13 days to 7.29 days, $p<0.01$ ). Treatment of a patient suffering from schizophrenia with oral antipsychotics produced costs of $9935.38 €$ (direct costs: 9498. $36 €$ ), while aripiprazole-depot generated costs of $4557.56 €$ (direct costs: $4449.83 €$ ) per patient for a one-year observation period. This resulted in total costs of 6,517,606,265.43€ for the oral antipsychotic treatment and 2,989,756,603.05€ for aripiprazole-depot treatment from the perspective of the German health care system. The results remained robust during sensitivity analysis, with aripiprazole-depot being the more cost-effective strategy.
\end{abstract}

Conclusions: The results suggest that aripiprazole-depot treatment for schizophrenia patients has major potential in terms of cost savings for the German statutory health insurance.

Keywords: Schizophrenia, Aripiprazole-depot, Long-acting injectable, Hospitalization, Budget-impact-analysis

\footnotetext{
* Correspondence: christoph.potempa@ifeg.de

Institute of Empirical Health Economics, Am Ziegelfeld 28, 51399 Burscheid Germany
}

(c) The Author(s). 2018 Open Access This article is distributed under the terms of the Creative Commons Attribution 4.0 International License (http://creativecommons.org/licenses/by/4.0/), which permits unrestricted use, distribution, and reproduction in any medium, provided you give appropriate credit to the original author(s) and the source, provide a link to the Creative Commons license, and indicate if changes were made. 


\section{Introduction}

Schizophrenia is estimated to affect 24 million individuals worldwide $\left(656,000-738,000^{1}\right.$ [1] in Germany considering a prevalence rate between $0.8 \%$ and $0.9 \%$ for patients between 18 and 65 years [2]). Total costs related to schizophrenia were estimated at $\$ 62.7$ billion in the US [3], and the total costs of schizophrenia and other psychotic disorders were estimated at 93.9 billion $€$ in Europe [4]. The annual direct costs of schizophrenia treatment per patient in Germany are estimated between $14,000-18,000 €[5]$ with hospitalizations and productivity losses as the main cost-driving factors. The annual total costs for schizophrenia in Germany are estimated between 4,400,000,000€ and 9,200,000,000€ [6].

The World Health Organization (WHO) estimates the costs of schizophrenia in western countries in a range between $1.6 \%$ and $2.6 \%$ of total health care expenditures. The social and economic consequences of mental illnesses are considerable high, with its treatment costs surpassing even heart diseases, cancer, and diabetes treatment [7].

The direct costs generated by schizophrenia comprise all resource consumption resulting from a treatment or therapy and are directly attributable to this disorder like medication costs, hospitalizations, outpatient and long-term care [8].

The indirect costs occur not directly in relation to the treatment of the disease but arise from the fact that schizophrenia is a very disabling disease. Schizophrenia is associated with productivity losses by the patient and the patient's family members. Work incapacities (temporary or definitive) and disability pensions due to schizophrenia can be very cost-intensive for the health care system [9]. Less reliable data are available on the indirect costs because resource- and productivity losses as a result of morbidity and mortality in the context of schizophrenia are more difficult to access [7].

Another reason for the high costs of schizophrenia arises from the fact that it is a chronic and lifelong condition. The resource consumption of patients with schizophrenia in Germany has not been evaluated in detail. Due to the cost-intensive characteristics of schizophrenia a reduction of these costs could result in significant savings from the perspective of the German statutory health insurance.

The reduction of the direct and indirect costs of schizophrenia can be realized by different procedures. Better and more cost-effective medications can be recognized as one of those solutions. Sustained release drugs (like aripiprazole-depot) are reported to have significantly higher adherence rates than traditional oral antipsychotics [10].

The findings on the effectiveness of aripiprazole-depot are also consistent with several clinical studies that associate reduced psychiatric hospitalization rates and durations with sustained release drugs compared to traditional oral anti-psychotic therapies [11].

Recent meta-analyses came to the conclusion that sustained release drugs are able to reduce the hospitalization risk significantly compared to oral antipsychotic treatments $[12,13]$. A total of 58 evaluated studies show that the reduction in hospitalization rates for LAIs (long-acting injectable) was 20.7 percentage points higher than that of OAs (oral antipsychotics) (LAIs $=56.2 \%$ vs. OAs $=35.5 \%$, $P=0.023)$ [13].

The main objectives of this study can be summarized in two sub goals:

- Examine whether aripiprazole-depot treatment reduces the hospitalization rates and other cost driving factors compared to oral medication treatment for schizophrenia.

- Compare conceivable benefits and cost-savings (including direct and indirect costs) for the German statutory health insurance for both observation phases based on several variables of resource consumption over a one-year observation time frame.

\section{Methods}

\section{Study design and observation group}

This study was carried out as a single armed, non-interventional pre-post comparison study with a subsequent BIA to assess hospitalization rates in patients with schizophrenia treated with oral antipsychotics followed by a treatment with aripiprazole-depot in a community setting in Germany. The study consisted of two retrospectively reported treatment-phases. The retrospective documentation period started in May 2016 and had been finalized in November 2016.

The required sample size was estimated at $n=116$ to provide $80 \%$ power to detect a statistically significant difference of $11 \%$ between the hospitalization rate during the aripiprazole-depot treatment period (Phase B) and a recently published hospitalization rate for patients being treated with oral antipsychotics (38.1\%) from the mentioned study [11]. A dropout rate of $10 \%$ was assumed, which resulted in a required total sample-size of $n=129$ patients (Fig. 1).

A total of 6994 physicians were contacted. The total number of recruited centers (psychiatrists) was 67 (from which 28 (41.8\%) were active with the 39 (58.2\%) remaining centers counting as dropouts due to lack of transmitted patient data). Data for 136 patients was collected at the end of the inclusion phase by a total of 28 psychiatrists in Germany. The database was closed on November the 30th 2016. 4 of those 136 patients (2.9\%) were excluded from further analysis because of not meeting the inclusion criteria (18-65 years) of the study. 
Data was documented for two different phases. During the six-month observation period phase A the patients schizophrenia was treated with traditional oral antipsychotics. With the end of phase A the treatment was switched to aripiprazole-depot for six subsequent months. The observation periods both had a duration of six months each, regardless whether the treatment itself lasted longer than six months. This condition ensured comparability between both treatment phases. Variables from the following three categories were retrieved during data collection phase and were considered in the statistical analyses:

- Socio-demographic and administrative parameters

- Indication- and efficacy based parameters

- Parameters of resource-utilization

\section{Study eligibility}

Eligible patients $(n=132)$ were aged between 18 and 65 years with a schizophrenia diagnosis consistent with the International Statistical Classification of Diseases and Related Health Problems (ICD-10). Patients also needed to have been prescribed an oral anti-psychotic treatment in the six months prior to the switch to aripiprazoledepot, which had to be documented for six subsequent months.

Eligible patients also required a change in treatment for any reason, with the specific reason being reported by the investigator (e.g. insufficient efficacy, insufficient compliance, side effects). This change in treatment represents no intervention (compared to RCTs) because of the retrospective character of the study. Eligibility was determined retrospectively by the investigator, which explains the low amount of patients that failed to meet the inclusion criteria of the study (4 patients).

Subjects with an ICD-10 diagnosis other than schizophrenia (2 patients with schizoaffective disorder) were included in the analysis because of being reported in multiple categories and having a schizophrenia diagnosis as well.

Some patients (the amount differed between variables) were excluded from several statistical procedures because of item-non-response or contradictions concerning the extent of some of the reported outcome variables. Hospitalized days for example or days with work incapacities were very occasionally reported for longer time-frames than the actual observation time-period (six months per phase).

\section{Endpoints}

The primary endpoint was the psychiatric hospitalization rate (defined as the proportion of patients with $\geq 1$ psychiatric hospitalization) which was assessed and compared between the oral anti-psychotic treatment period (months 1-6) and the aripiprazole-depot treatment period (months 7-12).

Additionally secondary efficacy and endpoint comparisons were compared between both treatment periods including the number of schizophrenia episodes, the mean duration of hospitalizations, the number of emergency treatments (emergency physicians and emergency services), the frequency and duration of day-clinic-visits as well as the number of psychiatrist visits and productivity losses in form of days of sick leave.

\section{Statistical analysis}

Comparisons between hospitalization rates during the retrospective oral anti-psychotic treatment period (months 1-6) and the aripiprazole-depot treatment period (months 7-12) were assessed using Wilcoxon signed rank tests with a statistical significance at an alpha level of 0.05 (two-sided) after using Kolgomorov-Smirnov tests to check the observed empirical distributions for normality.

Additionally the hospitalization rate of patients during the treatment with aripiprazole-depot was compared with the hospitalization rates conducted by a recent study by Kane et al. [11] using a one-sided binomial test.

\section{Budget-impact-analysis}

The BIA is a specific form of health economic evaluation, which evaluates the financial consequences of new technologies in a specific health care system [14]. The analysis form is increasingly required in national reimbursement procedures and can also be used for resource or budget planning [15].

In this investigation, a BIA was performed to estimate the annual budget impact of two different treatment options for schizophrenia. The analysis referred to the context of German health care, was performed from the perspective of the German statutory health insurance and considered direct and indirect costs of schizophrenia to deliver an overview on the resource utilization and the related costs of patients suffering from schizophrenia. It should be noted here that productivity losses in Germany only become relevant from the point of view of the statutory health insurances starting with the sixth week of sick leave. Therefore, the direct costs were evaluated separately in order to avoid misunderstandings regarding the direct and indirect costs of schizophrenia.

\section{Results}

\section{Demographics}

Demographics and baseline characteristics for the 132 patients that received treatment with aripiprazole-depot after the switch from oral antipsychotics are reported in Table 1 . The majority of patients were female $(n=71 /$ $132,53.8 \%)$ and the mean age was $37.89(\mathrm{sd}=10.54)$ years with a mean age at schizophrenia diagnosis of 
Table 1 Demographics

\begin{tabular}{ll}
\hline Measure & \\
\hline Age, mean (SD), years & 37.81 (10.54) \\
Male/female, $\mathrm{n}$ & $60 / 71$ \\
Body weight, mean (SD), kg & 84.94 (19.61) \\
Body height, mean (SD), cm & 171.05 (8.56) \\
BMl, mean (SD), kg/m² & 28.96 (6.17) \\
Age at schizophrenia diagnosis (SD), years & 30.55 (8.67) \\
Employed / unemployed / retired / education & 24 / 39 / 51/ 9 / 9 \\
/ NA, $\mathrm{n}$ & \\
Single / married / widowed / other /NA, n & 80 / 43 / 3 / 5 / 1 \\
\hline
\end{tabular}

30.55 years $(\mathrm{sd}=8.67) .92 .4 \%$ of the patients were insured statutory $(n=122 / 132)$ while 72 of these patients reported to have a co-payment exemption.

\section{Primary efficacy end-point: Total psychiatric hospitalization rates}

Psychiatric hospitalization rates (number of psychiatric hospitalizations $\geq 1$ ) in patients who were treated with aripiprazole-depot (Fig. 2) were significantly lower compared to the retrospective hospitalization rates (months 1-6) when treated with oral antipsychotics.

The treatment with aripiprazole-depot also significantly reduced the mean number of hospitalizations (0.63 hospitalizations per patient during phase A vs. 0.16 hospitalizations per patient during phase $\mathrm{B}, p<0.001$ ) and the mean number of hospitalized days (27.39 $(\mathrm{sd}=41.37)$ days in phase A vs. $5.56(\mathrm{sd}=20.70)$ days in phase $\mathrm{B}, \mathrm{p}<0.001$ ).

\section{Secondary efficacy end-points}

The days of sick leave (resulting in a productivity loss) were examined for the employed subsample only. This restriction was justified by the irrelevance of days of sick leave in terms of contribution to the indirect costs of schizophrenia and reduced the sample size to $n=24$ patients for this specific analysis. The mean number of days of sick leave during phase A was 40.06 compared to
15.80 days in phase $B$. This difference was not significant $(p=0.169)$.

The mean number of schizophrenia episodes was 2.58 $(\mathrm{sd}=2.91)$ episodes per patient in the first six months during the treatment with oral antipsychotics, and 0.41 $(\mathrm{sd}=0.76)$ for the second six month period with aripiprazole-depot treatment $(p<0.001)$.

The treatment change caused no significant reduction of the number of psychiatrist visits (10.57 to $7.73, p=0.07$ ) but instead there was a significant reduction of the mean stay in day-clinics and psychiatric institute ambulances (PIAs) (46.13 days (phase A) compared to 7.29 days (phase B), $p<0.01$ ).

\section{Cost parameters}

Cost savings were calculated from the perspective of the German statutory health insurance. The cost-relevant data for both treatment periods were derived from the cost variables in Table 2 and used in the BIA.

Both direct and indirect costs were considered. All the cost components were assessed in monetary units.

Table 3 shows per case costs of $9935.38 €$ for patients during oral-medication and $4557.56 €$ during aripiprazoledepot treatment. This suggests potential cost-savings of $5377.82 €$ per schizophrenia patient. $63,83 \%(1562.49 €)$ of the total oral medication costs are caused by aripiprazole in its oral form. Even without consideration of these costs caused by oral aripiprazole prescriptions, the total costs per unit stay are significantly lower in the depot-treatment phase. The direct costs (without productivity losses) were also lower for the aripiprazole-depot study-period (9498.36 $€$ vs. $4449.83 €)$.

Table 4 provides a breakdown of the total costs for the total study sample $(n=132)$ which were calculated under the assumption of a $100 \%$ substitution of schizophrenia patients with both treatment options. In this context, it should be noted that aripiprazole-depot is not a broad-based treatment option and is primarily prescribed for individuals with a history of non-adherence. Therefore, the projections in Table 4 must be interpreted as approximate estimates because a $100 \%$ substitution of aripiprazole-depot represents an unrealistic scenario.

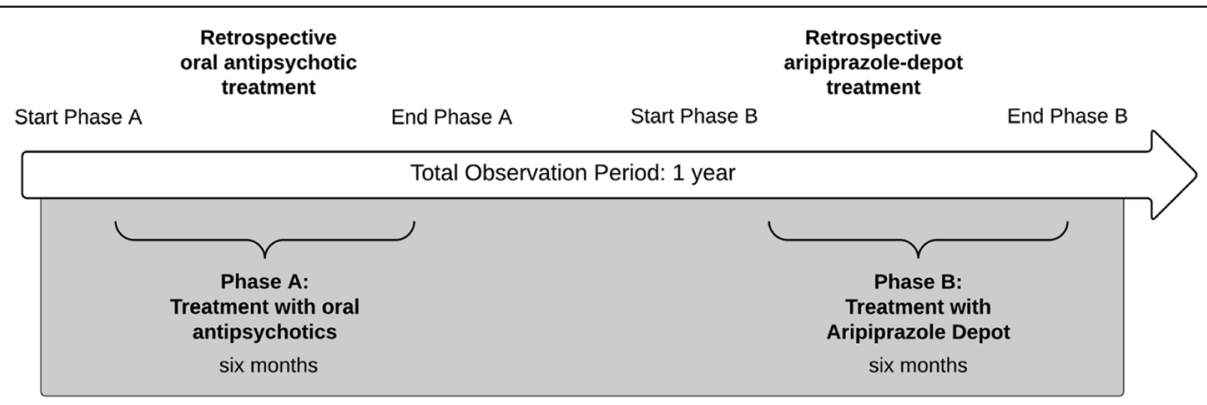

Fig. 1 Study design and time frame 




Fig. 2 Total Hospitalization rates following the switch to aripiprazole-depot (Phase B) with the same patients treated with oral antipsychotics (Phase A). $P$ value derived from Wilcoxon signed rank test

Treatment with aripiprazole-depot resulted in lower total costs compared to the oral medication treatment period $(601,597.37 €$ vs $1,311,469.55 €)$. This result remained similar also without consideration of the productivity losses (indirect costs) and distinctly supports the treatment with aripiprazole-depot. Hospitalizations and medications costs appear to be the main cost driving factors. During the treatment with aripiprazole-depot the reduced hospitalization-rates as well as the reduced number of hospitalized days resulted in significant cost savings compared to Phase A $(170,021.33 €$ vs. $746,168.65 €)$.

Other relevant cost driving factors were the productivity losses, the services (EBM / GOÄ) by the psychiatrists and the non-drug-interventions. Aripiprazole-depot remained the more cost-effective strategy for every single cost factor if medication costs were combined. ${ }^{2}$
Considering a prevalence rate between $0.8 \%$ and $0.9 \%$ [2] aripiprazole-depot treatment resulted in total costs between $2,989,756,603.05 €$ and 3,363,476,178.43€ compared to total costs between 6,517,606,265.43€ and $7,332,307,048.61 €$ (oral medication) for the total population of schizophrenia patients in Germany. ${ }^{3}$

\section{Sensitivity analyses}

In order to examine the robustness of the results, a univariate sensitivity analysis was conducted for this BIA. For this purpose, one parameter is varied while all other parameters are kept constant. This way, the influence of specific variables on the outcome can be quantified. The resulting transition probabilities of the BIA were varied by the standard deviation (mean $\pm \mathrm{sd}$ ) of the cost-relevant variable. Cost uncertainty was varied by the cost-range for the cost-relevant variable. ${ }^{4}$

Table 2 Cost Parameters and Sources

\begin{tabular}{lll}
\hline Treatment & Source & Base rate \\
\hline Productivity loss (days of sick leave) & Hannoveraner Konsens [18] & $90 €$ \\
EBM $^{3}$ Services & EBM (Einheitlicher Bewertungsmaßstab) [19] & $* *$ \\
GÖ̈4 Services & GOÄ (Gebührenordnung für Ärzte) [20] & $120 €-360 €^{*}$ \\
Days in day-clinics / PIA & BAG Psych. [21] & $* *$ \\
EBM Services during hospitalization & EBM [19] & $* *$ \\
GOÄ Services during hospitalization & GOÄ [20] & $90 €-385 €^{*}$ \\
Hospital days & InEK PEPP / BBFW (GKV Spitzenverband) [22] & $13.37 €$ \\
Emergency Services & EBM (Einheitlicher Bewertungsmaßstab) [19] & $171 €$ \\
Emergency Physician & DGINA [23] & $* *$ \\
Non-drug-interventions & Various sources & $515.97 €$ \\
Aripiprazole-depot injections & Rote Liste 2016 & $* *$ \\
Medication & Rote Liste 2016 & $* *$ \\
Medication (pre-existing conditions) & Rote Liste 2016 & $*$ \\
\hline
\end{tabular}

* Cost range considered in univariate sensitivity analyses, ${ }^{* *}$ Costs vary by specific service / medicine

${ }^{3}$ Doctors' Fee Scale within the Statutory Health Insurance Scheme

${ }^{4}$ Fee regulations for doctors within private Health Insurance Scheme 
Table 3 Costs per unit $(n=132)$

\begin{tabular}{lll}
\hline Treatment & oral medication & $\begin{array}{l}\text { aripiprazole- } \\
\text { depot }\end{array}$ \\
\hline Productivity loss (days of sick leave) & $437.02 €$ & $107.73 €$ \\
EBM Services & $314.37 €$ & $286.18 €$ \\
GOÄ Services & $28.64 €$ & $11.98 €$ \\
Days in day-clinics / PIA & $121.21 €$ & $63.64 €$ \\
EBM Services during hospitalization & $78.36 €$ & $26.02 €$ \\
GOÄ Services during hospitalization & $4.67 €$ & $1.29 €$ \\
Hospital days & $5652.79 €$ & $1288.04 €$ \\
Emergency Services & $3.13 €$ & $0.61 €$ \\
Emergency Physician & $12.88 €$ & $1.30 €$ \\
Non-drug-interventions & $373.42 €$ & $103.79 €$ \\
Aripiprazole-depot injections & $0.00 €$ & $2325.57 €$ \\
Medication & $2447.80 €$ & $103.35 €$ \\
Medication & $\left(885.31 €^{*}\right)$ & \\
(pre-existing conditions) & $461.07 €$ & $238.07 €$ \\
Total & & \\
Direct Costs (without productivity losses) & $9498.36 €$ & $4449.83 €$
\end{tabular}

* without the costs for aripiprazole (oral medication)

The following parameters were varied by using the subsequent values for each of both treatment options:

Probability of occurrence of:

- Productivity loss (days of sick leave) $\bigcirc$ [oral antipsychotics: $\min =10.44 ; \max =69.68$; mean $=40.06$; $\mathrm{sd}=29.62]$ $\bigcirc$ [aripiprazole-depot: $\min =0.00 ; \max =40.47$; mean $=15.80 ; \mathrm{sd}=24.67]$

- Days in day-clinics / PIAs

$\bigcirc$ [oral antipsychotics: $\min =0.66 ; \max =91.6$; mean $=46.13 ; \mathrm{sd}=45.47]$

$\bigcirc$ [aripiprazole-depot: $\min =0.00 ; \max =22.31$; mean $=7.29 ; \mathrm{sd}=15.02]$

- Number of hospital days

$\bigcirc$ [oral antipsychotics: $\min =0,00 ; \max =68.76$; mean $=27.39 ; \mathrm{sd}=41.37]$

$\bigcirc$ [aripiprazole-depot: $\min =0.00 ; \max =26.26$; mean $=5.56 ; \mathrm{sd}=20.70$ ]

- Number of emergency physician treatments

[oral antipsychotics: $\min =0.00 ; \max =2.21$; mean $=1.42$; $\mathrm{sd}=0.79]$

$\bigcirc$ [aripiprazole-depot: $\min =0.00 ; \max =3.00$; mean $=2.00$; $\mathrm{sd}=1.00$ ]

- Number of emergency service treatments $\bigcirc$ [oral antipsychotics: $\min =0.00 ; \max =4.91$; mean $=2.38$; $\mathrm{sd}=2.53]$

$\bigcirc$ [aripiprazole-depot: $\min =0.00 ; \max =3.00$; mean $=2.00$; $\mathrm{sd}=1.00$ ]

Additionally costs were varied due to non-standardized costs for the following variables:

- Days in day-clinics / PIAs

$O$ [both treatment options: $\min =240.00 €$; $\max =720.00 €]$

- Number of hospital days

$\bigcirc$ [both treatment options: $\min =90.00 € ; \max$ $=385.00 €]$

Table 4 Total costs $(n=132)$

\begin{tabular}{lll}
\hline Treatment & oral medication & aripiprazole-depot \\
\hline Productivity loss (days of sick leave) & $57,686.40 €$ & $14,220.00 €$ \\
EBM Services & $41.496,84 €$ & $37,775.82 €$ \\
GÖ̈ Services & $3.780,70 €$ & $1581.62 €$ \\
Days in day-clinics / PIA & $16,000.00 €$ & $8400.00 €$ \\
EBM Services during hospitalization & $10,344.07 €$ & $3434.13 €$ \\
GÖ̈ Services during hospitalization & $616.66 €$ & $170.07 €$ \\
Hospital days & $746,168.65 €$ & $170,021.33 €$ \\
Emergency Services & $413.67 €$ & $80.22 €$ \\
Emergency Physician & $1699.74 €$ & $171.00 €$ \\
Non-drug-interventions & $49,291.59 €$ & $13,699.69 €$ \\
Aripiprazole-depot injections & $0.00 €$ & $306,975.32 €$ \\
Medication & $323,109.37 €\left(116,860.96 €^{*}\right)$ & $13,642.75 €$ \\
Medication (pre-existing conditions) & $60,861.86 €$ & $31,425.42 €$ \\
Total & $1,311,469.55 €(1,105,221.14 € *$ & $601,597.37 €$ \\
Direct Costs (without productivity losses) & $1,253,783.15 €$ & $587,377.37 €$ \\
\hline
\end{tabular}

* without the costs for aripiprazole (oral medication) 
The results of the BIA remained robust to the modification of all seven uncertain cost-factors and probabilities during sensitivity analyses, with aripiprazole-depot being the more cost-effective strategy, with hospitalizations as the main cost-driving factor.

Costs for medication, psychiatrist services and non-druginterventions were kept constant in the sensitivity analyses due to their characteristics (already being calculated as the sum of different medications, services and interventions), as no meaningful interpretable standard deviation could be calculated for the total costs.

The results of the sensitivity analysis are displayed in Table 5 (per case and total sample population) and Table 6 (extrapolation for the population of schizophrenia patients in Germany).

The base case analysis was already introduced in Table 3 and Table 4 and is used as a reference value for the variations in the sensitivity analysis. The results of the two therapy options remained robust to the removal of transitional probabilities and cost uncertainties. The probability of occurrence of days of sick leave (productivity loss) was varied by its own standard deviation and led to cost ranges of $9612.25 €$ to $10,258.50 €$ (oral medication) and $4449.83 €$ to $4725.76 €$ (aripiprazole-depot) per patient. The days in day clinics were varied in two different ways. The variation of the occurrence-probability by its own standard deviation led to a cost range of $9894.16 €$ to $10,056.59 €$ (oral medication) and $4493.92 €$ to $4621.19 €$ (aripiprazole-depot). Additionally the costs were varied for the day-clinic / PIA days because there is no fixed cost-value for these services. The variation of costs led to cost-ranges of $9862.16 €$ and $10,250.53 €$ (oral medication) and $4493.92 €$ to 4723.01 $€$ (aripiprazole-depot).

The parameters hospital days and the associated costs of hospital days revealed the largest cost range and were identified as the parameters with the largest impact on parameter uncertainty. The variation of the occurrenceprobability of the number of hospital days led to cost ranges of $4282.58 €$ to $18,473.38 €$ (oral medication) and $3269.52 €$ to $9352.96 €$ (aripiprazole-depot). Because of the fact that the hospitalization-costs are not standardized and differ significantly, costs for hospitalizations were varied as well. The cost-variation led to a cost range of $4282.58 €$ to $29,351.33 €$ for oral medication treatment and $3269.52 €$ to $12,843.47 €$ for aripiprazole-depot treatment. The parameters for emergency physician treatments / services achieved the lowest cost range. Therefore they achieved the smallest impact on parameter uncertainty.

Table 5 Results of the univariate sensitivity analysis (Sample Population)

\begin{tabular}{|c|c|c|c|c|}
\hline \multirow[t]{2}{*}{ Treatment } & \multicolumn{2}{|c|}{$\varnothing$ Per case costs $(n=132)$} & \multicolumn{2}{|c|}{$\varnothing$ Total costs $(n=132)$} \\
\hline & oral medication & aripiprazole-depot & oral medication & aripiprazole-depot \\
\hline Base case analysis & $9935.38 €$ & $4557.56 €$ & $1,311,469.55 €$ & $601,597.37 €$ \\
\hline \multicolumn{5}{|c|}{ Probability of occurence of productivity loss (days of sick leave) } \\
\hline $\min$ & $9612.25 €$ & $4449.83 €$ & $1,268,816.75 €$ & $587,377.37 €$ \\
\hline $\max$ & $10,258.50 €$ & $4725.76 €$ & $1,354,122.35 €$ & $623,800.37 €$ \\
\hline \multicolumn{5}{|c|}{ Probability of occurence of days in day-clinics / PIA } \\
\hline $\min$ & $9894.16 €$ & $4493.92 €$ & $1,306,029.55 €$ & $593,197.37 €$ \\
\hline $\max$ & $10,056.59 €$ & $4621.19 €$ & $1,327,469.55 €$ & $609,997.37 €$ \\
\hline \multicolumn{5}{|c|}{ Cost uncertainty of days in day-clinics / PIA } \\
\hline $\min$ & $9862.16 €$ & $4493.92 €$ & $1,301,805.55 €$ & $593,197.37 €$ \\
\hline $\max$ & $10,250.53 €$ & $4723.01 €$ & $1,353,069.55 €$ & $623,437.37 €$ \\
\hline \multicolumn{5}{|c|}{ Probability of occurence / number of hospital days } \\
\hline $\min$ & $4282.58 €$ & $3269.52 €$ & $565,300.90 €$ & $431,576.04 €$ \\
\hline $\max$ & $18,473.38 €$ & $9352.96 €$ & $2,438,486.61 €$ & $1,234,590.43 €$ \\
\hline \multicolumn{5}{|c|}{ Cost uncertainty of hospital days } \\
\hline $\min$ & $4282.58 €$ & $3269.52 €$ & $565,300.90 €$ & $431,576.04 €$ \\
\hline $\max$ & $29,351.33 €$ & $12,843.47 €$ & $3,874,375.90 €$ & $1,695,338.54 €$ \\
\hline \multicolumn{5}{|c|}{ Probability of occurence of emergency physician } \\
\hline $\min$ & $9922.50 €$ & $4556.26 €$ & $1,309,769.81 €$ & $601,426.37 €$ \\
\hline $\max$ & $9948.04 €$ & $4561.82 €$ & $1,313,141.33 €$ & $602,160.27 €$ \\
\hline \multicolumn{5}{|c|}{ Probability of occurence of emergency services } \\
\hline $\min$ & $9932.24 €$ & $4556.95 €$ & $1,311,055.89 €$ & $601,517.15 €$ \\
\hline $\max$ & $10,037.63 €$ & $4573.63 €$ & $1,324,966.93 €$ & $603,718.86 €$ \\
\hline
\end{tabular}


Table 6 Results of the univariate sensitivity analysis (Schizophrenia Population in Germany)

\begin{tabular}{|c|c|c|c|c|}
\hline \multirow[t]{2}{*}{ Treatment } & \multicolumn{2}{|c|}{$\varnothing$ Total costs $(n=656,000)$} & \multicolumn{2}{|c|}{$\varnothing$ Total costs $(n=738,000)$} \\
\hline & oral medication & aripiprazole-depot & oral medication & aripiprazole-depot \\
\hline Base case analysis & $6,517,606,265.43 €$ & $2,989,756.603 .05 €$ & $7,332,307,048.61 €$ & $3,363,476,178.43 €$ \\
\hline \multicolumn{5}{|c|}{ Probability of occurence of productivity loss (days of sick leave) } \\
\hline $\min$ & $6,305,634,774.52 €$ & $2,919,087,512.14 €$ & $7,093,839,121.34 €$ & $3,283,973,451.16 €$ \\
\hline $\max$ & $6,729,577,756.34 €$ & $3,100,098,784.87 €$ & $7,570,774,975.88 €$ & $3,487,611,132.98 €$ \\
\hline \multicolumn{5}{|c|}{ Probability of occurence of days in day-clinics / PIA } \\
\hline $\min$ & $6,490,571,113.92 €$ & $2,948,011,148.51 €$ & $7,301,892,503.16 €$ & $3,316,512,542.07 €$ \\
\hline $\max$ & $6,597,121,416.95 €$ & $3,031,502,057.60 €$ & $7,421,761,594.07 €$ & $3,410,439,814.80 €$ \\
\hline \multicolumn{5}{|c|}{ Cost uncertainty of days in day-clinics / PIA } \\
\hline $\min$ & $6,469,579,113.92 €$ & $2,948,011,148.51 €$ & $7,278,276,503.16 €$ & $3,316,512,542.07 €$ \\
\hline $\max$ & $6,724,345,659.37 €$ & $3,098,294,784.87 €$ & $7,564,888,866.79 €$ & $3,485,581,632.98 €$ \\
\hline \multicolumn{5}{|c|}{ Probability of occurence of hospital days } \\
\hline $\min$ & $2,809,374,180.43 €$ & $2,144,802,139.41 €$ & $3,160,545,952.99 €$ & $2,412,902,406.84 €$ \\
\hline $\max$ & $12,118,539,502.25 €$ & $2,296,772,366.69 €$ & $13,633,356,940.03 €$ & $2,583,868,912.52 €$ \\
\hline \multicolumn{5}{|c|}{ Cost uncertainty of hospital days } \\
\hline $\min$ & $2,809,374,180.43 €$ & $2,144,802,139.41 €$ & $3,160,545,952.99 €$ & $2,412,902,406.84 €$ \\
\hline $\max$ & $19,254,474,180.43 €$ & $8,425,318,806.08 €$ & $21,661,283,452.99 €$ & $9,478,483,656.84 €$ \\
\hline \multicolumn{5}{|c|}{ Probability of occurence of emergency physician treatments } \\
\hline $\min$ & $6,509,159,072.70 €$ & $2,988,906,784.87 €$ & $7,322,803,956.79 €$ & $3,362,520,132.98 €$ \\
\hline $\max$ & $6,525,914,487.19 €$ & $2,992,554,070.32 €$ & $7,341,653,798.09 €$ & $3,366,623,329.11 €$ \\
\hline \multicolumn{5}{|c|}{ Probability of occurence of emergency services } \\
\hline $\min$ & $6,515,550,461.82 €$ & $2,989,357,933.96 €$ & $7,329,994,269.55 €$ & $3,363,027,675.70 €$ \\
\hline $\max$ & $6,584,684,146.81 €$ & $3,000,299,790.32 €$ & $7,407,769,665.16 €$ & $3,375,337,264.11 €$ \\
\hline
\end{tabular}

Table 5 shows these per case costs for the sample population $(n=132)$ while Table 6 extrapolates these costs under consideration of the estimated prevalence-rate of schizophrenia in Germany. The calculations for the German total population were made for both prevalence rates $(0.8 \%$ / 0.9\%). Table 7 shows the results under the assumption of a substitution of aripiprazole-depot between 4 and $12 \%$ for schizophrenia treatment [16].

\section{Discussion / limitations}

The pre-post comparison study demonstrated significantly lower psychiatric hospitalization rates for patients switched from an oral antipsychotic therapy to aripiprazole-depot, as well as significantly less non-drug interventions and a reduced amount of productivity losses due to days of sick leave. The reduction of the hospitalization rates remained significant when being compared to a recent study and its reported hospitalization rate [11].

The BIA evaluated the costs and financial consequences of both schizophrenia treatments for the German health care system from the perspective of the German statutory health insurance. Treatment with oral-medication causes total costs of $6,517,606,265.43 €$, which are significantly higher than the total costs for treatment with aripiprazole-depot $(2,989,756,603.05 €)$. Considering the six-month observation period per treatment, these extrapolated costs for oral schizophrenia were significantly higher that the estimated annual schizophrenia costs in Germany (between 4,400,000,000 $€$ and 9,200,000,000€ per year) [6].

Based on the study data, treatment with aripiprazoledepot is clearly more cost-effective than treatment with oral-medication for schizophrenia, which is mainly associated with less and shorter hospitalizations. These results support recent analyses which reported reduced health-care related costs for sustained release drugs compared to oral antipsychotics [17].

Regardless of the hospitalization-costs, aripiprazoledepot resulted in lower costs on all economical variables, including total drug-acquisition costs, which is an unexpected result considering the often reported greater initial acquisition costs associated with sustained release drugs [11]. These results can be put into perspective by the fact that $63,83 \%$ of the total medication costs in the oral treatment phase are caused by aripiprazole in its oral form. 
Table 7 Results of the univariate sensitivity analysis (estimated market-share)

\begin{tabular}{|c|c|c|c|c|}
\hline \multirow[t]{2}{*}{ Treatment } & \multicolumn{2}{|c|}{ Total costs $(n=656,000 * 0.04)$} & \multicolumn{2}{|c|}{ Total costs $(n=738,000 * 0.12)$} \\
\hline & oral medication & aripiprazole-depot & oral medication & aripiprazole-depot \\
\hline Base case analysis & $260.704 .250,62 €$ & $119.590 .264,12 €$ & $879.876 .845,83 €$ & 403.617.141,41€ \\
\hline \multicolumn{5}{|c|}{ Probability of occurence of productivity loss } \\
\hline $\min$ & $252.225 .390,98 €$ & $116.763 .500,49 €$ & $851.260 .694,56 €$ & $394.076 .814,14 €$ \\
\hline $\max$ & $269.183 .110,25 €$ & $124.003 .951,39 €$ & $908.492 .997,11 €$ & $418.513 .335,96 €$ \\
\hline \multicolumn{5}{|c|}{ Probability of occurence of days in day-clinics / PIA } \\
\hline $\min$ & $259.622 .844,56 €$ & $117.920 .445,94 €$ & $876.227 .100,38 €$ & $397.981 .505,05 €$ \\
\hline $\max$ & $263.884 .856,68 €$ & $121.260 .082,30 €$ & $890.611 .391,29 €$ & $409.252 .777,78 €$ \\
\hline \multicolumn{5}{|c|}{ Cost uncertainty of days in day-clinics / PIA } \\
\hline $\min$ & $258.783 .164,56 €$ & $117.920 .445,94 €$ & $873.393 .180,38 €$ & $397.981 .505,05 €$ \\
\hline $\max$ & $268.973 .826,37 €$ & $123.931 .791,39 €$ & $907.786 .664,02 €$ & $418.269 .795,96 €$ \\
\hline \multicolumn{5}{|c|}{ Probability of occurence of hospitalized days } \\
\hline $\min$ & $112.374 .967,22 €$ & $85.792 .085,58 €$ & $379.265 .514,36 €$ & $289.548 .288,82 €$ \\
\hline $\max$ & $484.741 .580,09 €$ & $245.421 .612,30 €$ & $1.636 .002 .832,80 €$ & $828.297 .941,53 €$ \\
\hline \multicolumn{5}{|c|}{ Cost uncertainty of hospitalized days } \\
\hline $\min$ & $112.374 .967,22 €$ & $85.792 .085,58 €$ & $379.265 .514,36 €$ & $289.548 .288,82 €$ \\
\hline $\max$ & $770.178 .967,22 €$ & $337.012 .752,24 €$ & 2.599.354.014,36€ & $1.137 .418 .038,82 €$ \\
\hline \multicolumn{5}{|c|}{ Probability of occurence of emergency physician treatments } \\
\hline $\min$ & $260.366 .362,91 €$ & $119.556 .271,39 €$ & $878.736 .474,82 €$ & $403.502 .415,96 €$ \\
\hline $\max$ & $261.036 .579,49 €$ & $119.702 .162,81 €$ & $880.998 .455,77 €$ & 403.994.799,49€ \\
\hline \multicolumn{5}{|c|}{ Probability of occurence of emergency services } \\
\hline $\min$ & $260.622 .018,47 €$ & $119.574 .317,36 €$ & $879.599 .312,35 €$ & $403.563 .321,08 €$ \\
\hline $\max$ & $263.387 .365,87 €$ & $120.011 .991,61 €$ & $888.932 .359,82 €$ & $405.040 .471,69 €$ \\
\hline
\end{tabular}

Possible limitations of the pre-post comparison study are a potential selection bias of patients and psychiatrists due to missing randomization as well as reduced sample-sizes on variables that were analysed for the employed subsample only. There was no inclusion of a control group as the patients served as their own control. The pre-post study design additionally can be prone to biases because of time-changing context factors.

Additionally the sample sizes were comparatively low, especially with regard to the employed sub-population, as the study provides a pilot project to investigate the care situation of schizophrenia patients in Germany. The results should be tested and confirmed by future projects with significantly larger case numbers. These future research efforts should also focus more on the side effects of schizophrenia.

Some cost-variables were accessed by estimations. These estimations were documented and considered during sensitivity analyses, but despite that are inferior to empirically accessed cost data.

In that regard, the collection of additional economic data of the affected patients could complement the study data efficiently. Larger sample-sizes could help to enable inferential statistical procedures for subpopulations (like employed people only) and randomization in a prospective research design could lead to a reduction of selection bias. The present evaluation is also solely dealing with costs to the health insurance system and the patient himself and does not take into account other costs in terms of work productivity or time lost or burden to family members. The sensitivity-analysis which considered the estimated market-share of aripiprazole-depot was based on data for the UK [16]. Future analyses should take the German shares into account to reduce the bias generated due to the comparison of two different health-systems.

Another potential limitation is the fact that productivity losses were analyzed for the employed sample population only, which resulted in a reduced sub-population. It was not considered if the unemployment was caused by the schizophrenia. In this case, productivity losses and the associated costs would be systematically underestimated in the study results. 


\section{Conclusion}

The BIA contributes relevant data on the discussion of schizophrenia treatment in Germany that are in line with recent publications on the health-economic advantages of sustained release drugs like aripiprazole-depot compared to oral antipsychotic medication.

The results suggest that schizophrenia treatment with aripiprazole-depot instead of an oral antipsychotic treatment leads to significant cost reductions from the perspective of the German statutory health insurance.

The cost-saving potential is primarily explained by less and shorter hospitalizations combined with a smaller amount of productivity losses and a significant reduction of the mean stay in day-clinics and psychiatric institute ambulances (PIAs) for patients with aripiprazole-depot treatment.

\section{Endnotes}

${ }^{1}$ Calculation based on a total population of 82.800 .000

${ }^{2}$ Total medication costs were calculated by the sum of aripiprazole-depot injections (which were not used in Phase A), concomitant drugs and medications due to pre-existing conditions.

${ }^{3}$ These costs were calculated for the duration of the observation period (6 months). For annual costs the amount has to be doubled.

${ }^{4}$ Cost ranges for days in day-clinics / PIA and hospitalized days were used because of the absence of fixed costs on these variables and were accessed from the literature.

\section{Abbreviations}

BIA: Budget Impact Analysis; EBM: Einheitlicher Bewertungsmaßstab Doctors' Fee Scale within the Statutory Health Insurance Scheme; GOÄ: Gebührenordnung für Ärzte - Fee regulations for doctors within private Health Insurance Scheme; ICD-10: International Statistical Classification of Diseases and Related Health Problems; LAl: Long acting injectable; OA: Oral antipsychotics; PIA: Psychiatric institute ambulances; RCT: Randomized controlled trial; WHO: World Health Organization

\section{Acknowledgements}

Not applicable.

\section{Funding}

This study was sponsored by Otsuka Pharmaceutical Development \& Commercialization, Inc. and Lundbeck $\mathrm{GmbH}$. The sponsor was involved in the study design but not for analysis and interpretation of the data, in the writing of the report and manuscript.

\section{Availability of data and materials}

The data that support the findings of this study are available, but restrictions apply to the availability of these data, which were used under license for the current study, and so are not publicly available. Data are however available from the authors upon reasonable request and with permission of Otsuka Pharmaceutical Development \& Commercialization, Inc. and Lundbeck $\mathrm{GmbH}$.

\section{Authors' contributions}

CP analyzed and interpreted the study data and was a major contributor in writing the manuscript. RR was a major contributor in writing the manuscript. All the authors contributed to the critical revision of the manuscript, and gave approval of the final version of the manuscript.

\section{Publisher's Note}

Springer Nature remains neutral with regard to jurisdictional claims in published maps and institutional affiliations.

Received: 26 June 2018 Accepted: 16 November 2018

Published online: 23 November 2018

\section{References}

1. Bundesamt S. Bevölkerung in Deutschland voraussichtlich auf 82,8 Millionen gestiegen. Pressemitteilung vom 27 Januar 2017 2017;33(17).

2. Gaebel W, Wölwer W. Gesundheitsberichterstattung des Bundes Heft 50. Berlin: Rober Koch Institut; 2010.

3. Wu EQ, Birnbaum HG, Shi L, Ball DE, Kessler RC, Moulis M, et al. The economic burden of schizophrenia in the United States in 2002. J Clin Psychiatry. 2005:66(9):1122-9.

4. Gustavsson A, Svensson M, Jacobi F, Allgulander C, Alonso J, Beghi E, et al. Cost of disorders of the brain in Europe 2010. Eur Neuropsychopharmacol. 2011;21(10):718-79.

5. Konopka A, Klingberg S, Wittorf A, König H. Die Kosten der Schizophrenie in Deutschland-Ein systematischer Literaturüberblick. Psychiatr Prax. 2009;36(5):211-8.

6. Kissling W, Höffler J, Seemann U, Müller $P$, Rüther $E$, Ue T, et al. Die direkten und indirekten Kosten der Schizophrenie. Fortschritte der Neurologie. Psychiatrie. 1999;67(01):29-36.

7. Barbato A. Schizophrenia and public health, Division of Mental Health and Prevention of Substance Abuse. World Health Organization; 1998.

8. Tajima-Pozo K, de Castro Oller MJ, Lewczuk A, Montañes-Rada F. Understanding the direct and indirect costs of patients with schizophrenia. F1000Research. 2015:4.

9. Kortmann L-M, Müller D, Simic D, Civello D, Stock S. Erwerbsminderung bei Schizophrenie-eine empirische Analyse der Folgelasten. Psychiatr Prax. 2016

10. Hasan A, Falkai P, Wobrock T. Schizophrenia WTfoTGf. Guidelines for biological treatment of schizophrenia, part 2: update 2012 on the long-term treatment of schizophrenia and management of antipsychotic-induced side effects. WJ Biol Psychiatry. 2013;14:2-44.

11. Kane JM, Zhao C, Johnson BR, Baker RA, Eramo A, McQuade RD, et al. Hospitalization rates in patients switched from oral anti-psychotics to aripiprazole once-monthly: final efficacy analysis. J Med Econ. 2015;18(2):145-54

12. Kishimoto T, Nitta M, Borenstein M, Kane JM, Correll CU. Long-acting injectable versus oral antipsychotics in schizophrenia: a systematic review and meta-analysis of mirror-image studies. The Journal of clinical psychiatry. 2013:74(10):957-65.

13. Lafeuille M-H, Dean J, Carter V, Duh MS, Fastenau J, Dirani R, et al. Systematic review of long-acting injectables versus oral atypical antipsychotics on hospitalization in schizophrenia. Curr Med Res Opin. 2014:30(8):1643-55.

14. Lauterbach KW. Gesundheitsökonomie, Management und Evidence-based Medicine: Handbuch für Praxis. Schattauer Verlag: Politik und Studium; 2010.

15. Sullivan SD, Mauskopf JA, Augustovski F, Caro JJ, Lee KM, Minchin M, et al. Budget impact analysis - principles of good practice: report of the ISPOR 2012 budget impact analysis good practice II task force. Value Health. 2014;17(1):5-14.

16. NHS. Aripiprazole (Abilify Maintena ${ }^{\oplus}$ ) prolonged release IM (depot) injection for maintenance treatment of patients with schizophrenia stabilised on oral aripiprazole. London Medicines Evaluation Network Review. 2014:1-5.

17. Lin J, Wong B, Offord S, Mirski D. Healthcare cost reductions associated with the use of LAI formulations of antipsychotic medications versus oral among patients with schizophrenia. The journal of behavioral health services \& research. 2013:40(3):355-66.

18. von der Schulenburg J-MG, Greiner W, Jost F, Klusen N, Kubin M, Leidl R, et al. Deutsche Empfehlungen zur gesundheitsökonomischen Evaluation-dritte und aktualisierte Fassung des Hannoveraner Konsens. Gesundheitsökonomie \& Qualitätsmanagement. 2007;12(05):285-90.

19. Bundesvereinigung DK. Einheitlicher Bewertungsmaßstab (EBM). Stand: 1. Quartal. 2016:2016.

20. Hess R, Krimmel L. Gebührenordnung für Ärzte (GOÄ). Deutscher ÄrzteVerlag, Köln. 1996

21. Krankenhäuser BPBdTP. Psychiatrische Institutsambulanzen in Deutschland Kassel 2008 
22. InEK. PEPP-Entgeldkatalog Version 2016. Institut für das Entgeldsystem im Krankenhaus. 2016.

23. Haas C, Larbig M, Schöpke T, Lübke-Naberhaus K, Schmidt C, Brachmann M. Gutachten zur ambulanten Notfallversorgung im Krankenhaus-

Fallkostenkalkulation und Strukturanalyse. Hamburg: Management Consult Kestermann GmbH (MCK; 2015.

Ready to submit your research? Choose BMC and benefit from:

- fast, convenient online submission

- thorough peer review by experienced researchers in your field

- rapid publication on acceptance

- support for research data, including large and complex data types

- gold Open Access which fosters wider collaboration and increased citations

- maximum visibility for your research: over $100 \mathrm{M}$ website views per year

At BMC, research is always in progress.

Learn more biomedcentral.com/submissions 